\title{
Heat-fluid Coupling Simulation of Wet Friction Clutch
}

\author{
Tengjiao Lin ${ }^{1, a^{*}}$, Qing Wang ${ }^{1, b}$, Quancheng Peng ${ }^{1, c}$ and Yan Xie ${ }^{1, d}$ \\ ${ }^{1}$ State Key Laboratory of Mechanical Transmission, Chongqing University, China

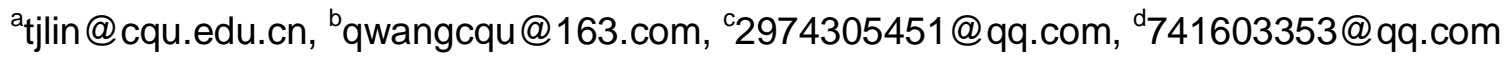

\begin{abstract}
Keywords: Clutch, Heat-fluid coupling, Temperature distribution, Finite element method Abstract. In order to estimate the heating value of wet multi-plate friction clutch at idle state, the heat-fluid coupling simulation of oil way fluid field is carried out. Taking oil way of clutch as research object, dynamic mesh technique is adopted to implement the dynamic simulation about fluid field in disc separation process. Based on the actual state of oil way at the end of disc separation process of disengaging clutch, simulation model about heat-fluid coupling of oil way is established through combining energy conservation law with shear heating theory of oil film. Then the paper simulates the temperature filed of lubricant, and researches the influence principle of drive shaft speed and lubricant flow on lubricant temperature. Results shows: the maximum temperature and exit temperature of lubricant increase gradually as idling speed increases; and they will decrease gradually as inlet flow increases.
\end{abstract}

\section{Introduction}

In marine clutch-reversible gearbox, pair-mounted wet multi-plate friction clutches are in engaging and disengaging condition respectively. While clutch is idling in disengaging condition, there is great speed difference between friction disc and dual plate. Oil film among discs will then emerge fluid kinetic energy under shear effect of oil film, and heat quantity changes with it. Clutch therefore generates heat, which will truly affect wet multi-plate friction clutch.

Currently, research about heat generating problem of friction clutch focuses on temperature filed calculation of friction disc, etc. Through heat conduct model of friction disc, numerical simulation about heat conduct process of friction disc is realized via FEM [1,2,3]. Along with gradual maturation of CFD technology, heat-fluid coupling simulation of complicated fluid field is acceptable by combining computer numerical simulation with graphic display, which involves vortex pipe, refrigerator and so on [5]. However, study about heat-fluid coupling of oil way fluid field for friction clutch is still rare.

This paper sets up simulation model about heat-fluid coupling of oil way on the basis of actual oil way situation obtained by dynamic simulation of disc separation process for clutch. Adopting RNG $k-\varepsilon$ vortex model, the oil way fluid field and temperature field are calculated. Influence principle of drive shaft speed and lubricant flow on lubricant temperature is also researched, after which heating value prediction of idling clutch is realized.

\section{Governing Equations of Heat-fluid Coupling}

In the analysis of lubricant heat-fluid coupling, RNG k- $\varepsilon$ vortex model is employed, and governing equations comprised of mass conversation equation, momentum conversation equation and energy conversation equation are also involved. There the mass conversation equation and momentum conversation equation are used for solving the pressure and velocity of lubricant, and the temperature of lubricant can be obtained by combining the energy conversation equation. 


$$
\begin{aligned}
& \frac{\partial \rho}{\partial t}+\frac{\partial(\rho u)}{\partial x}+\frac{\partial(\rho v)}{\partial y}+\frac{\partial(\rho w)}{\partial z}=0 \\
& \left\{\begin{array}{l}
\frac{\partial(\rho u)}{\partial t}+\operatorname{div}(\rho u u)=\operatorname{div}(\mu \operatorname{grad} u)-\frac{\partial p}{\partial x}-\rho g_{x} \\
\frac{\partial(\rho v)}{\partial t}+\operatorname{div}(\rho v u)=\operatorname{div}(\mu \operatorname{grad} v)-\frac{\partial p}{\partial y}-\rho g_{y} \\
\frac{\partial(\rho w)}{\partial t}+\operatorname{div}(\rho w u)=\operatorname{div}(\mu \operatorname{grad} w)-\frac{\partial p}{\partial z}-\rho g_{z}
\end{array}\right.
\end{aligned}
$$

where the Eq. 1 is mass conversation equation, and the Eq. 2 is momentum conversation equation, in which $\operatorname{grad}()=\partial() / \partial x+\partial() / \partial y+\partial() / \partial z ; \rho$ is lubricant density; $\mu$ is dynamic viscosity; $p$ is lubricant pressure.

In the RNG k- $\varepsilon$ turbulence model, the turbulent kinetic energy equation (Eq. 3) and the turbulent dissipation rate equation (Eq. 4) for incompressible fluids can be described as:

$$
\begin{aligned}
& \frac{\partial k}{\partial t}+\sum_{i=1}^{3} \frac{\partial\left(u_{i} k\right)}{\partial x_{i}}=\sum_{i=1}^{3} \frac{1}{\rho} \frac{\partial}{\partial x_{i}}\left[\left(\mu+\frac{\mu_{t}}{\sigma_{k}}\right) \frac{\partial k}{\partial x_{i}}\right]+\frac{1}{\rho} G_{k}-\varepsilon \\
& \frac{\partial \varepsilon}{\partial t}+\sum_{i=1}^{3} \frac{\partial\left(u_{i} \varepsilon\right)}{\partial x_{i}}=\sum_{i=1}^{3} \frac{1}{\rho} \frac{\partial}{\partial x_{i}}\left[\left(\mu+\frac{\mu_{t}}{\sigma_{\varepsilon}}\right) \frac{\partial \varepsilon}{\partial x_{i}}\right]+\frac{1}{\rho} \frac{C_{1 \varepsilon}^{*} \varepsilon}{k} G_{k}-C_{2 \varepsilon} \frac{\varepsilon^{2}}{k}
\end{aligned}
$$

where $k$ is turbulent kinetic energy; $\varepsilon$ is turbulent dissipation rate; $\mu_{t}$ is turbulent viscosity; $G_{k}$ is the item of turbulent kinetic energy caused by gradient of average velocity; $C_{1 \varepsilon}^{*}$ is the correction coefficient of constant $C_{1 \varepsilon}$ of RNG $k-\varepsilon$ turbulence model.

Conversation law of fluid energy can be presented as: the energy increasing rate of micro-element in fluid channel equals to the sum of net heat flux entering micro-element and work done on micro-element by volume force and area force. Thus energy conservation law of micro-element about clutch lubricant is Eq. 5.

$$
\frac{\partial\left(\rho c_{p} T\right)}{\partial t}+\frac{\partial\left(\rho v_{x} c_{p} T\right)}{\partial x}+\frac{\partial\left(\rho v_{y} c_{p} T\right)}{\partial y}+\frac{\partial\left(\rho v_{z} c_{p} T\right)}{\partial z}=\frac{\partial}{\partial x}\left(k \frac{\partial T}{\partial x}\right)+\frac{\partial}{\partial y}\left(k \frac{\partial T}{\partial y}\right)+\frac{\partial}{\partial z}\left(k \frac{\partial T}{\partial z}\right)+Q_{v}
$$

where $c_{p}$ is specific heat of lubricant, $T$ is lubricant temperature and $Q_{v}$ is viscous dissipation.

Combining Eq. (1), Eq. (2), Eq. (3), Eq. (4) and Eq. (5), heat-fluid coupling of clutch lubricant can be analyzed.

\section{Fluid Dynamic Simulation of Clutch}

The Structure of Clutch. Fig. 1 is the structure of wet multi-disc friction clutch. When the clutch is idling in disengaging operation, the clearances between discs are filled with oil ejected from four nozzle groups, and friction discs will separate automatically by influence of lubricant which then flows out through five oil exit. Initially, we assume that all discs are evenly distributed, and all clearances between discs are the same.

Fluid Dynamic Simulation. The lubricant CD40 is used in clutch, and the density and kinematic viscosity are $880 \mathrm{~kg} / \mathrm{m}^{3}$ and $43.4 \times 10^{-6} \mathrm{~m}^{2} / \mathrm{s}$ at $58^{\circ} \mathrm{C}$. The solid and finite element models of oil structure are established in ANSYS. Fig. 2 shows the finite element models of oil structure and boundary conditions, the inlet flow is $110 \mathrm{~L} / \mathrm{min}$ and the outlet pressure is an atmospheric pressure. The wall of shell is stationary, the fluid near wall is processed with wall function, and steel discs and gears are rotating clockwise by $1213 \mathrm{r} / \mathrm{min}$.

Stationary solutions of turbulence model of the separation process are obtained by the 
semi-implicit algorithm with coupled equations of pressure and velocity. The dynamic simulation of the separation process is conducted by using dynamic mesh method.

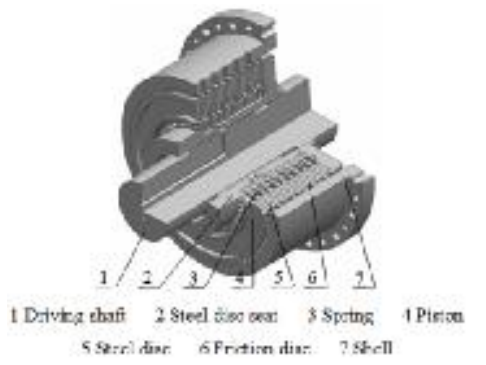

Fig.1. The structure of clutch
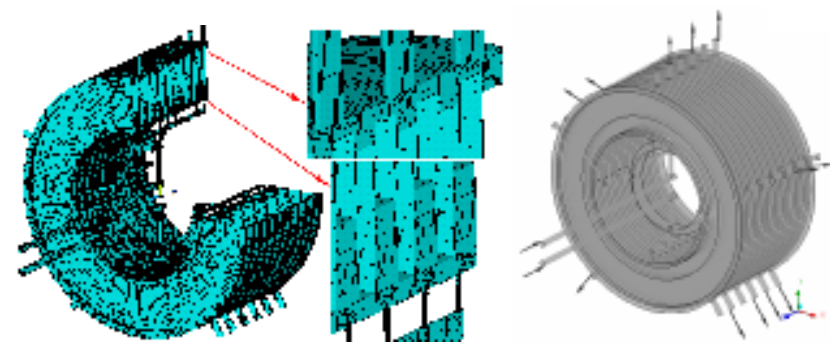

Fig.2. Finite element model of oil structure

\section{Heat-fluid Coupling Analysis of Clutch}

Convective Heat Transfer Coefficient. Lubricant flow can be divided into three categories: laminar flow, transitional laminar flow and vortex flow. In these different flow categories, convective heat transfer coefficient is related to Prandtl number and Reynolds number. Reynolds number and Prandtl number can be solved by Eq. 6:

$$
\operatorname{Re}=v_{d} / v_{f}, \operatorname{Pr}=\rho v_{f} C / \lambda
$$

Eq. 7 shows Nusselt number criterion equation(Sieder-Tate) and convective heat transfer coefficient equation.

$$
N u=0.027 \operatorname{Re}^{0.8} \operatorname{Pr}^{1 / 3}\left(\mu_{f} / \mu_{w}\right)^{0.14}, h=N u \cdot \lambda / d
$$

where $\mu_{f}$ and $\mu_{w}$ are fluid dynamic viscosity determined by fluid average temperature and wall temperature respectively.

Heat-fluid Coupling Analysis. Oil way obtained by dynamic simulation of disc separation process is used to build lubricant heat-fluid coupling model for idling clutch. In CFX, total thermal model which takes thermal quantity changes caused by fluid kinetic energy into consideration is employed. This subsection analyzes the lubricant temperature distribution of oil way while idling speed of drive shaft is $1213 \mathrm{r} / \mathrm{min}$ and housing speed is $0 \mathrm{r} / \mathrm{min}$. Boundary conditions of lubricant way include: (1) fixing wall temperature is $50^{\circ} \mathrm{C}$, convective heat transfer coefficient is $1266.24 \mathrm{~W} \cdot\left(\mathrm{m}^{2} \cdot \mathrm{K}\right)^{-1} ;(2)$ an atmospheric pressure for exit; (3) inlet oil temperature is $58^{\circ} \mathrm{C}$, and inlet volumetric flow is $110 \mathrm{~L} / \mathrm{min}$; (4) housing wall is static, and walls at clutch gear and dual plate rotate clockwise at a speed of $1213 \mathrm{r} / \mathrm{min}$.

Fig. 3 is Lubricant pressure contour. Lubricant pressure loses greatly at the corners of oil way and the nozzles, and maximum oil pressure occurs at inlet hole. Fig. 4 is Lubricant velocity contour. Lubricant velocity changes greatly at shape mutation places such as oil-separating holes and nozzles, and after lubricant flows into oil-separating holes, flow rate increases as channel diameter decreases.

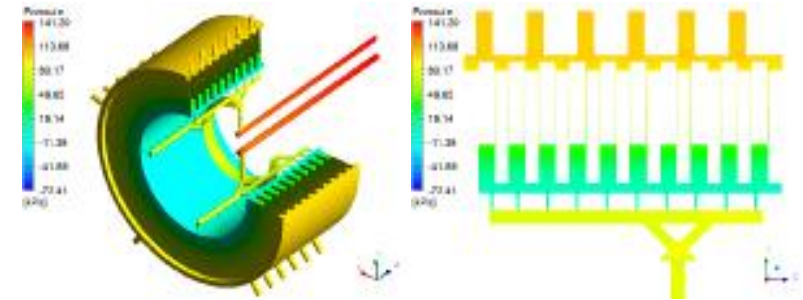

Fig. 3. Lubricant pressure contour

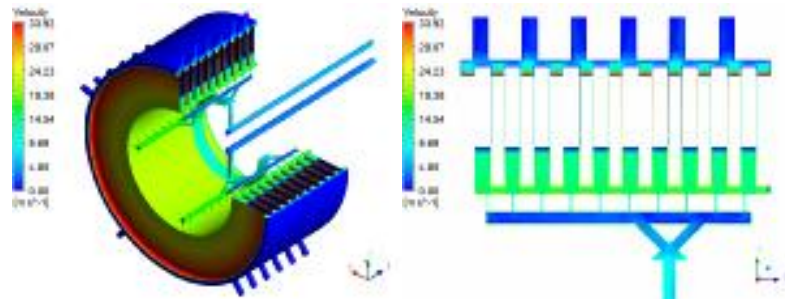

Fig. 4. Lubricant velocity contour

Temperature contour of oil way obtained by heat-fluid coupling simulation is shown in Fig. 5 
while clutch idling speed is $1213 \mathrm{r} / \mathrm{min}$. From the figure, linear velocity difference increases as diameter gets bigger, and then shear heating of oil film gets more serious. In a word, oil temperature increases as diameter gets bigger, and it will decrease to some extent as there exists oil outlet and convective heat transfer of clutch housing at the outer diameter.
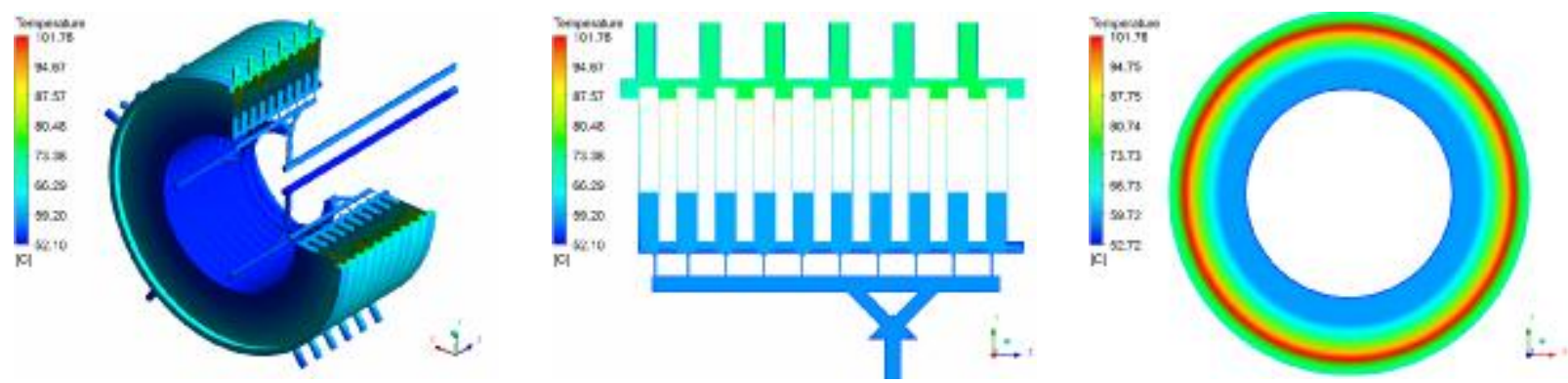

Fig. 5. Lubricant temperature contour

\section{Influence of Applying Working Condition on Lubricant Temperature}

Influence of Drive Shaft Speed on Lubricant Temperature. This subsection mainly analyzes temperature distribution of oil way at different rotation rate while clutch inlet flow is $110 \mathrm{~L} / \mathrm{min}$. Table 1 shows convective heat transfer coefficient with different rotational speed.

Table 1. Convective heat transfer coefficient with different rotational speed

\begin{tabular}{cccccc}
\hline Rotational speed $n_{\mathrm{e}} / \mathrm{r} \cdot \mathrm{min}^{-1}$ & 834 & 1000 & 1213 & 1400 & 1600 \\
\hline $\begin{array}{c}\text { Convective heat transfer coefficient } \\
\mathrm{h} / \mathrm{W} \cdot\left(\mathrm{m}^{2} \cdot \mathrm{K}\right)^{-1}\end{array}$ & 936.49 & 1082.79 & 1266.24 & 1419.70 & 1580.08 \\
\hline
\end{tabular}

Temperature contour of clutch lubricant at different drive shaft speed is given in Fig. 6. From the figure, the maximum clutch oil temperature increases gradually as rotation rate gets bigger. The maximum temperature and the outlet temperature of lubricant between discs are given in Table 2 . From the table, as a comprehensive effect of film shear heating and heat taken away by lubricant, clutch outlet temperature increases gradually as speed gets bigger.

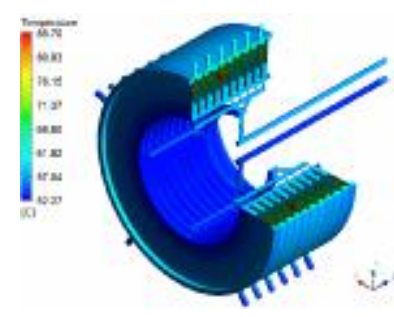

(a) $834 \mathrm{r} / \mathrm{min}$

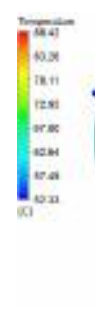

(b) $1000 \mathrm{r} / \mathrm{min}$

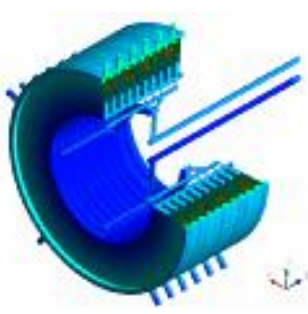

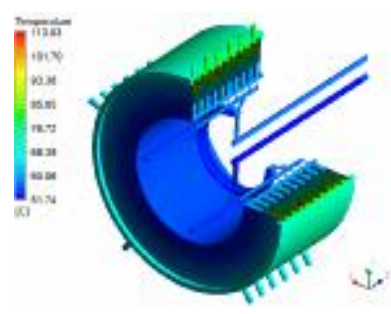

(c) $1400 \mathrm{r} / \mathrm{min}$

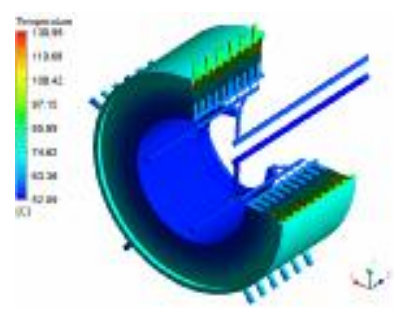

(d) $1600 \mathrm{r} / \mathrm{min}$

Fig.6. Lubricant temperature contour of the clutch with different rotational speed

Table 2. The temperature with different rotational speed

\begin{tabular}{cccccc}
\hline Rotational speed $n_{\mathrm{e}} / \mathrm{r} \cdot \min ^{-1}$ & 834 & 1000 & 1213 & 1400 & 1600 \\
\hline Maximum temperature $T_{\max }{ }^{\circ} \mathrm{C}$ & 85.7 & 88.42 & 101.76 & 110.03 & 130.95 \\
Outlet temperature $T_{\text {out }} /{ }^{\circ} \mathrm{C}$ & 64.65 & 67.87 & 74.85 & 82.01 & 94.61 \\
\hline
\end{tabular}

Influence of Inlet Flow on Lubricant Temperature. This section analyzes temperature 
distribution of oil way at different inlet flow while clutch idling speed is $1213 \mathrm{r} / \mathrm{min}$. Fig. 7 shows temperature contour of clutch lubricant at different inlet flow. From the figure, the maximum clutch oil temperature decreases gradually as inlet flow gets bigger. Table 3 shows the maximum temperature and the outlet temperature of lubricant between discs. From the table, clutch outlet oil temperature decreases gradually as inlet flow gets bigger as a comprehensive effect of film shear heating and heat taken away by lubricant.

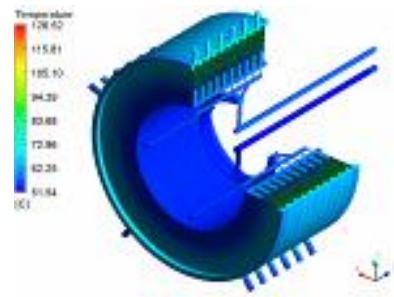

(a) $70 \mathrm{~L} / \mathrm{min}$

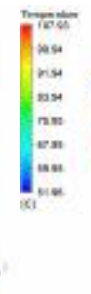

(b) $90 \mathrm{~L} / \mathrm{min}$

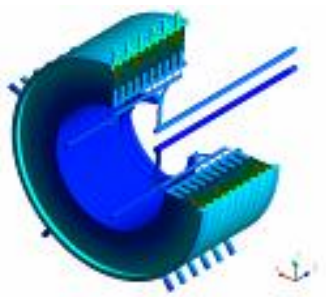

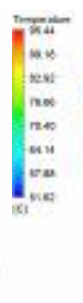

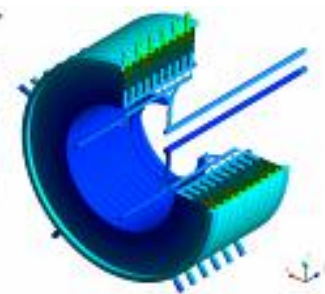

(c) $130 \mathrm{~L} / \mathrm{min}$
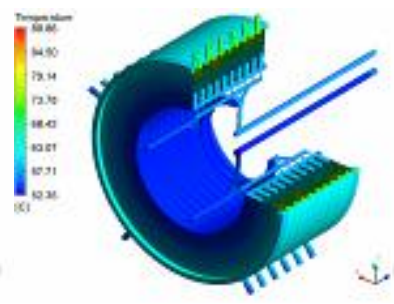

(d) $150 \mathrm{~L} / \mathrm{min}$

Fig.7. Lubricant temperature contour of the clutch with different inlet flow

Table 3. Lubricant temperature with different inlet flow

\begin{tabular}{cccccc}
\hline Inlet flow rate $Q / \mathrm{L} \cdot \mathrm{min}^{-1}$ & 70 & 90 & 110 & 130 & 150 \\
\hline Maximum temperature $T_{\max } /{ }^{\circ} \mathrm{C}$ & 126.52 & 107.93 & 101.76 & 95.44 & 89.86 \\
Outlet temperature $T_{\text {out }} /{ }^{\circ} \mathrm{C}$ & 76.83 & 75.49 & 74.85 & 73.71 & 73.16 \\
\hline
\end{tabular}

\section{Conclusions}

1) Lubricant temperature is basically unchangeable before lubricant flows between friction disc, and the maximum temperature is $101.76^{\circ} \mathrm{C}$ which occurs at oil film between discs. Different oil film has different temperature as each oil film has different thickness, which means clutch idling heating mainly comes from lubricant shear between friction discs.

2) Through heat-fluid coupling analysis of oil way at different idling speed and different inlet flow, results show: the maximum lubricant temperature and the outlet temperature increase gradually as idling speed gets bigger; and they will decrease gradually as inlet flow gets bigger.

\section{Acknowledgement}

The authors are grateful for the financial support provided by the Fundamental Research Funds for the State Key Laboratory of Mechanical Transmission under Contract No. SKLMT-ZZKT-2012 MS 06.

\section{References}

[1] Yue Hong, Jin Liu, Yungeng Wang. Chinese Jouranl of Mechanical Engineering. 17 (2004) 102-106. (In Chinese)

[2] Jen T C, Nemecek D J. International Journal of Heat and Mass Transfer. 51 (2008) 1757-1769.

[3] Shuangmei Zhao, Hilmas G E, Dharani L R. Wear. 264 (2008) 1059-1068.

[4] Smith E, Pongjet P. International Communications in Heat and Mass Transfer. 35(2008) 937-947.

[5] Xiaobin Zhang, Zhihua Gan, Limin Qiu. Journal of Zhejiang University SCIENCE A. 9 (2008) 93-98. (In Chinese) 